MATEC Web of Conferences 33, 05009 (2015)

DOI: $10.1051 /$ matec conf/ 20153305009

(c) Owned by the authors, published by EDP Sciences, 2015

\title{
Shape memory behavior of Ni-Fe-Ga and Ni-Mn-Sn ribbons
}

\author{
E.Villa ${ }^{1, a}$, C.O Agilar-Ortiz ${ }^{2}$, PÁ Ivarez-Alonso ${ }^{3}$, J.P. Camarillo² , G.A. Lara-Rodriguez ${ }^{4}$, H. Flores-Zúñiga ${ }^{2}$, V.A.Chernenko ${ }^{5,6}$ \\ ${ }^{1}$ CNR IENI Unità di Lecco, 23900 Lecco, Italy \\ ${ }^{2}$ División de Materiales Avanzados, IPICYT, 78216 San Luis Potosí, Mexico \\ ${ }^{3}$ Departamento de Electricidad y Electrónica, Universidad del País Vasco (UPV/EHU), 48940 Leioa, Spain \\ ${ }^{4}$ Instituto de Investigaciones en Materiales, Universidad Nacional Autónoma de México, 04510 México D.F., México \\ ${ }^{5}$ Ikerbasque, Basque Foundation for Science, 48013 Bilbao, Spain \\ ${ }^{6}$ BCMaterials \& University of Basque Country (UPV/EHU), 48080 Bilbao, Spain
}

\begin{abstract}
Magnetic Shape Memory Alloys exhibit multifunctional properties due the martensitic transformation (MT) and accompanying changes in the magnetic subsystem. In this work, melt spun ribbons of nominal composition $\mathrm{Ni}_{55} \mathrm{Fe}_{16} \mathrm{Ga}_{29}$ (R1) and $\mathrm{Ni}_{50} \mathrm{Mn}_{40} \mathrm{Sn}_{10}$ (R2) have been produced and characterized by the calorimetric, thermomechanical, mechanical, microscopic and X-ray measurements. The effect of short annealing on MT and thermo-mechanical properties is rather modest and in the case of R2 enhances its brittleness. R1 and R2 as-spun ribbons show MT at $\mathrm{T}_{\mathrm{M}} \sim 340 \mathrm{~K}$ and $\sim 420 \mathrm{~K}$, respectively. The strain-stress curves for Ni-Fe-Ga in austenitic phase indicate good visco-elastic properties, although the superelastic effect was not well pronounced. Ni-Fe-Ga as-cast and annealed samples, as well as the as-cast Ni-Mn-Sn ribbons, present a shape memory effect with an anomalous contraction and elongation at low external stresses in the course of the forward and reverse MT, consequence of the compressive internal stresses.
\end{abstract}

\section{Introduction}

Shape Memory Alloys (SMAs) have been aim of an intense activity due to their peculiar multifunctional properties associated with the reversible first-order phase transition driven thermally or mechanically, called martensitic transformation (MT) [1].

Recently another interesting mechanic-caloric effect, the elastocaloric effect (ECE), has attracted more attention into these systems. ECE consists in the isothermal entropy change or adiabatic temperature change within a material as a consequence of applying/removing of a stress, and in some bulk alloys like $\mathrm{Cu}-\mathrm{Zn}-\mathrm{Al}, \mathrm{Ni}-\mathrm{Mn}-\mathrm{Sb}-$ $\mathrm{Co}$, and $\mathrm{Ni}-\mathrm{Mn}-\mathrm{Sn}(\mathrm{Cu})$ a notable ECE has been observed [2-5]. It is a well-known fact that thermo-mechanical characterization is the principal way to investigate the actuating properties of these materials and to indicate the possibility of an optimization of their functional performance. Handling with SMAs usually requires the preparation of either single crystals [6] or textured polycrystals to maximize their peculiar functional effects and, to some extent, avoid the problems related to the high brittleness of these materials. The melt-spinning is a frequently used cost effective way to produce SMAs in the form of the ribbons representing a ready material for the tensile applications, e.g., in solid state refrigeration. Recent studies of Ni-Fe-Al ribbons obtained by the meltspinning technique have shown an enhanced strength and considerable recoverable strain [7-8]. This technique

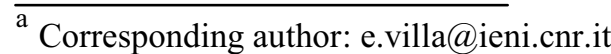

apparently presents interesting fabrication advantages, as it makes unnecessary plastic deformation routes to machine the material with the appropriate geometries, and the high temperature thermal treatment step usually needed to obtain single-phase alloy with given functional properties [9].

It should be emphasized that magnetically ordered SMAs, called magnetic shape memory materials, exhibit excellent ordinary shape memory characteristics (e.g., [25]). In this work, melt spun ribbons of the ferromagnetic SMA, $\mathrm{Ni}_{55} \mathrm{Fe}_{16} \mathrm{Ga}_{29}$ (at.\%), and metamagnetic SMA, $\mathrm{Ni}_{50} \mathrm{Mn}_{40} \mathrm{Sn}_{10} \quad$ (at.\%), have been prepared and characterized under reduced values of the external stress preferable for ECE applications. The effect of the short annealing on MT, elastic properties and shape memory effect has been also checked.

\section{Materials and methods}

Melt-spun ribbons with nominal compositions (at. \%) $\mathrm{Ni}_{55} \mathrm{Fe}_{16} \mathrm{Ga}_{29}$ (hereafter referred to as R1) and $\mathrm{Ni}_{50} \mathrm{Mn}_{40} \mathrm{Sn}_{10}$ (R2) were produced by a melt spinning technique, with a surface velocity of $20 \mathrm{~ms}^{-1}$ and $35 \mathrm{~ms}^{-1}$ for R1 and R2, respectively. The resulting ribbons were in the form of flakes from $\sim 10$ to $20 \mu \mathrm{m}$ thick and 0.9/1.9 $\mathrm{mm}$ wide for R1/R2. Polycrystalline master alloys were prepared from the pure elements by arc melting in $\mathrm{Ar}$ atmosphere. Mn was added to compensate the 
evaporation losses. Ribbons were studied in as-cast condition and after annealing at $773 \mathrm{~K}$ in inert atmosphere followed by water quenching. Annealing caused a strong embrittlement in the case of $\mathrm{R} 2$ ribbon that prevents its thermomechanical testing.

DSC analysis was performed by a Q100 TA Instruments calorimeter in the temperature range of $273 \mathrm{~K}-673 \mathrm{~K}$ and cooling/heating rate of $5 \mathrm{~K} / \mathrm{min}$. Characteristic MT temperatures, $\mathrm{M}_{\mathrm{S}}, \mathrm{M}_{\mathrm{F}}, \mathrm{A}_{\mathrm{S}}, \mathrm{A}_{\mathrm{F}}$, determined as the intersection of a baseline and the tangents to each peak, are listed in Table I. Microstructure and chemical composition were examined by a FEI/Philips XL30 FEG ESEM Scanning Electron Microscope (SEM) equipped with an x-ray energy dispersive spectroscopy (EDS) system. Measured composition of the samples is given in Table I. Crystal structure of the specimens was identified by powder X-ray diffraction (Bruker AXS model D8
Advance diffractometer, $\mathrm{Cu}-\mathrm{K} \alpha$ radiation). Le Bail analysis was performed using a FullProf Suite [10]. The static and dynamic mechanical characterization has been carried out by Dynamic Mechanical Analyzer DMA Q800 TA Instrument. A series of dependences "strain versus temperature" under constant load have been obtained for all the samples, with temperature rate of 5 $\mathrm{K} / \mathrm{min}$ and variable stress in the range 1-50 $\mathrm{MPa}$. The stress-strain curves were recorded at different temperatures in isothermal condition starting from high temperatures in a stress control mode with a stress rate of $2.5 \mathrm{MPa} / \mathrm{min}$. After each registered curve, the sample was heated (at zero load) above the $\mathrm{A}_{\mathrm{F}}$ temperature up to 473 $\mathrm{K}$ and $523 \mathrm{~K}$ for the Ni-Fe-Ga and Ni-Mn-Sn ribbons, respectively, to obtain all possible recovery of the residual deformation after the stress-strain process.

Table I. Chemical compositions, characteristic temperatures of MT ( $T_{\mathrm{M}}$ and $T_{\mathrm{A}}$ correspond to the temperatures of the DSC peak for the forward and reverse transformations, respectively), transformation hysteresis and enthalpy for the studied ribbons. Ribbon R1-A was annealed at $523 \mathrm{~K}$ for $10 \mathrm{~min}$.

\begin{tabular}{ccccccccccc}
\hline Ribbon & Composition & $\mathrm{T}_{\mathrm{M}}(\mathrm{K})$ & $\mathrm{M}_{\mathrm{S}}(\mathrm{K})$ & $\mathrm{M}_{\mathrm{F}}(\mathrm{K})$ & $\Delta \mathrm{H}(\mathrm{J} / \mathrm{g})$ & $\mathrm{T}_{\mathrm{A}}(\mathrm{K})$ & $\mathrm{A}_{\mathrm{S}}(\mathrm{K})$ & $\mathrm{A}_{\mathrm{F}}(\mathrm{K})$ & $\Delta \mathrm{H}(\mathrm{J} / \mathrm{g})$ & $\Delta \mathrm{T}(\mathrm{K})$ \\
\hline $\mathrm{R} 1$ & $\mathrm{Ni}_{55.1} \mathrm{Fe}_{16.2} \mathrm{Ga}_{28.7}$ & 349 & 359 & 337 & 5.1 & 361 & 350 & 371 & 4.2 & 12 \\
$\mathrm{R} 1-\mathrm{A}$ & $\mathrm{Ni}_{55.1} \mathrm{Fe}_{16.2} \mathrm{Ga}_{28.7}$ & 345 & 353 & 334 & 4.9 & 358 & 347 & 366 & 4.5 & 13 \\
$\mathrm{R} 2$ & $\mathrm{Ni}_{50.3} \mathrm{Mn}_{39.7} \mathrm{Sn}_{10.0}$ & 423 & 427 & 416 & 24.7 & 433 & 430 & 435 & 21.4 & 8 \\
\hline
\end{tabular}

\section{Results and discussion}

\subsection{Calorimetric analysis}

The results of DSC tests for both ribbons in as received and after annealing states are depicted in Fig.1. Both ribbons show good reproducibility of calorimetric measurements if heating/cooling proceeds up to a certain temperature, such as $500 \mathrm{~K}$ and $600 \mathrm{~K}$ for the R1 and R2 samples, respectively. Insets to Fig. 1 show that heating above these temperatures is accompanied by a start of the exothermic process consisting of the contributions from $\gamma$-phase precipitation, lattice defects rearrangements, and atomic ordering. Figure 1 shows that after annealing at $773 \mathrm{~K}$ for $1 \mathrm{~h}$. the DSC peak is becoming diffuse in the case of R1, while for R2 it is even a bit sharper. This reflects an importance of the different mechanisms responsible for such a behavior: in the case of R1 precipitation could be considered as the main mechanism, whereas an enhanced brittleness in the case of annealed R2 could indicate the defects influence and atomic ordering as the dominant factors. It is worth noting that the transition temperatures are slightly lower than those reported for bulk $\mathrm{Ni}_{50} \mathrm{Mn}_{40} \mathrm{Sn}_{10}$ alloy [10]. Wang et al. have proposed that such a reduction is associated with the existence of internal stress developed during the fast crystallization process [11]

Having in mind a very diffuse DSC peaks for the R1 sample, annealed at $773 \mathrm{~K}$ for $1 \mathrm{~h}$., we have performed a heat treatment for the as-cast Ni-Fe-Ga ribbons at $523 \mathrm{~K}$ for $10 \mathrm{~min}$. (a temperature close to that at which the aforementioned diffusion controlled process takes place).
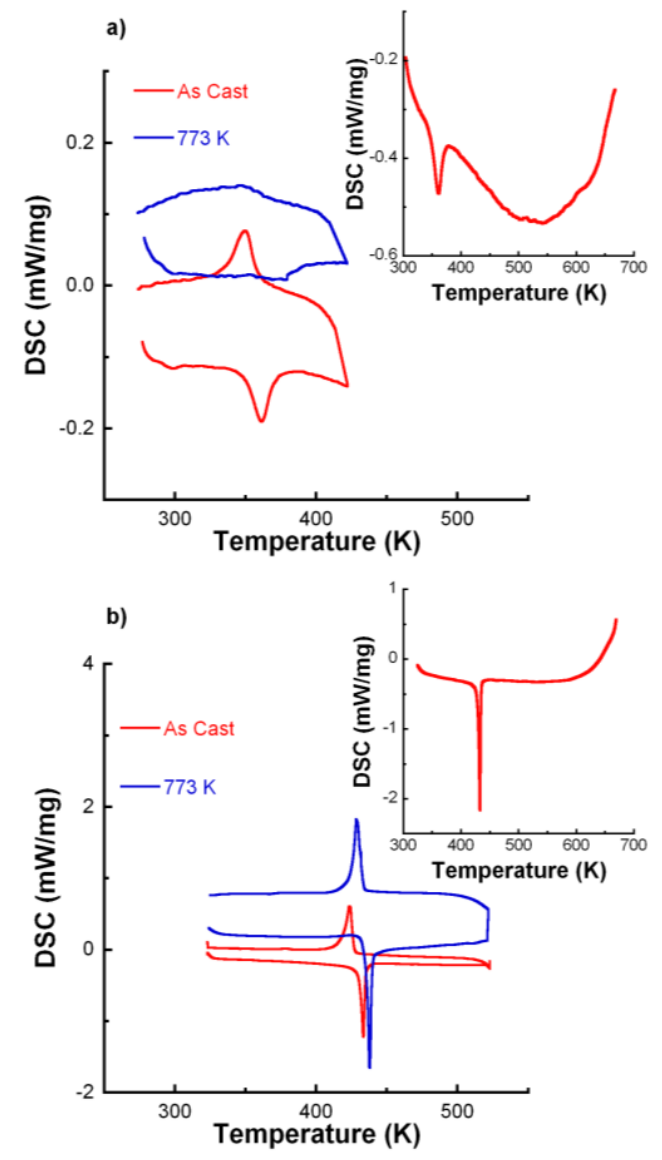

Figure 1. DSC graphs of Ni-Fe-Ga (a) and Ni-Mn-Sn (b) ribbons in the as-received and annealed at $773 \mathrm{~K}$ for $1 \mathrm{~h}$. states. Insets: heating runs up to $670 \mathrm{~K}$. 
Hereafter, this sample is called R1-A. DSC curves for R1-A (not shown) indicate a slight decrease of the characteristic MT temperatures (see Table I), but no changes in the thermal hysteresis $\left(\Delta T\right.$, defined as $A_{F}-$ $M_{S}$ ). These facts agree with a previous study of the annealing effect on the MT temperatures in such alloys [12].

\subsection{Crystal structure and microstructure}

Figures 2 (a) and (b) depict the X-ray powder diffraction pattern obtained at room temperature for the as-received $\mathrm{Ni}_{55.1} \mathrm{Fe}_{16.2} \mathrm{Ga}_{28.7}$ and $\mathrm{Ni}_{50.3} \mathrm{Mn}_{39.7} \mathrm{Sn}_{10.0}$ ribbons, respectively. As expected from the calorimetric analysis, $\mathrm{R} 1$ and R2 are in the martensite phase. All diffraction peaks for R1 were fitted to a monoclinic cell (space group $\mathrm{P} 2 / \mathrm{m}$ ) with a $5 \mathrm{M}$ superstructural lattice modulation, which has been previously observed in $\mathrm{Ni}_{2} \mathrm{FeGa}$ bulk alloys [13]. The cell parameters are: $a=4.19 \AA, b=5.51$ $\AA, c=20.53 \AA, \beta=88.73^{\circ}$. No secondary phases were detected, as normally observed in the bulk Ni-Fe-Ga alloys [14]. For R2, Bragg reflections correspond to an orthorhombic structure (space group P222) with 6layered modulation along the $a$-axis and cell parameters: $a=36.42 \AA, b=5.93 \AA, c=5.59 \AA$.

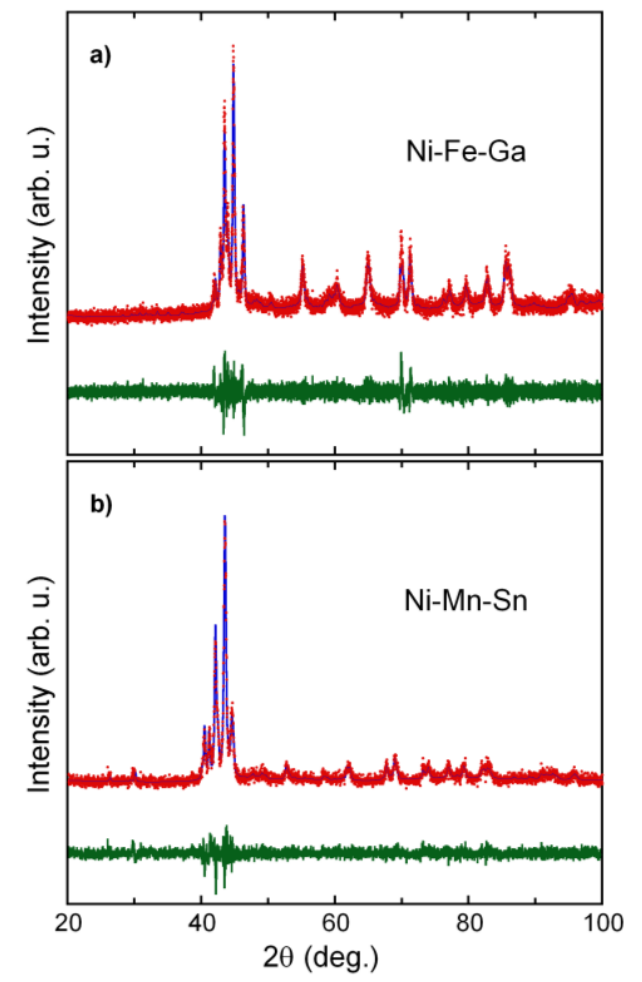

Figure 2. Observed (dots) and calculated (solid line) X-ray powder diffraction patterns of the as-cast Ni-Fe-Ga (a) and Ni$\mathrm{Mn}-\mathrm{Sn}$ (b) ribbons collected at room temperature.

Figures 3(a) and (b) show SEM images of the cross sections of the $\mathrm{Ni}-\mathrm{Fe}-\mathrm{Ga}$ and $\mathrm{Ni}-\mathrm{Mn}-\mathrm{Sn}$ ribbons respectively. Ni-Fe-Ga ribbons present typically misoriented grains of diameter about $4 \pm 1 \mu \mathrm{m}$. No gamma phase precipitates has been detected, in agreement with X-ray diffraction. Besides, on the free face (not shown), R1 presents a globular morphology with grain structure showing plates of twinned martensite variants with planar parallel interfaces. Similar results have been observed for R1-A. In the case of the specimens annealed at $773 \mathrm{~K}$ for $1 \mathrm{~h}$, no clear lamellar structure has been observed, which agrees with the fact that samples exhibit diffuse DSC peak at MT reflecting a small volume fraction of martensite formed.

The Ni-Mn-Sn ribbons exhibit an out-of-plane textured columnar structure, which is one of the reasons for its enhanced brittleness when stressed along the ribbon length. The ribbon surface shows a globular pattern incorporating grain structure and martensite lamellae superimposed. The average grain size across column (2.0 $\pm 0.5 \mu \mathrm{m})$ is definitely smaller compared with bulk alloys (50-300 $\mu \mathrm{m})$ [11]. The formation of columnar grains perpendicular to the ribbon plane is a microstructural signature of a fast nucleation/growth process along the whole ribbons cross-section during the cooling from the melt due to the highly anisotropic heat removal typical of this fabrication technique.

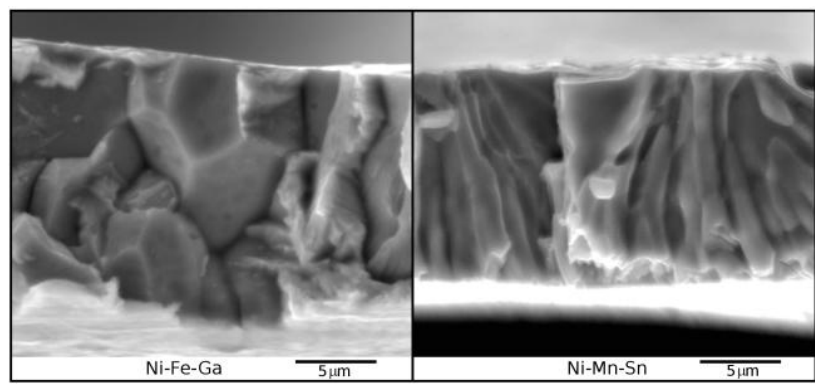

Figure 3. SEM images of the cross-section of the $\mathrm{Ni}_{55} \mathrm{Fe}_{16} \mathrm{Ga}_{29}$ (left) and $\mathrm{Ni}_{50.3} \mathrm{Mn}_{39.7} \mathrm{Sn}_{10.0}$ (right) samples.

\subsection{Mechanical and thermomechanical properties}

\subsubsection{Ni-Fe-Ga as-cast and annealed ribbons}

Figure 4 (a) shows the tensile stress-strain, $\sigma-\varepsilon$, curves for the $\mathrm{Ni}-\mathrm{Fe}-\mathrm{Ga}$ as-cast ribbons at selected temperatures below and above MT temperature. The curves show an initial elasticity and a non-linear dependence in the entire temperature range. The mechanism behind the nonlinear deformation process is different depending on the temperature: (i) below MT the plastic-like deformation of specimen results mainly from the twin variant redistribution; (ii) at temperatures within martensitic hysteresis, the $\sigma-\varepsilon$ curves present a pronounced hysteresis and a change in the slope, which indicates that the applied stress induces MT, giving rise to the two-phase state, martensite and austenite, with different elastic modulus; and (iii) above MT, the samples in austenitic state accumulate some amount of the plastic deformation due to the activation of dislocation mechanism. It is worth noting that, for the temperatures between $\mathrm{M}_{\mathrm{S}}$ and $\mathrm{A}_{\mathrm{F}}$, the stresses required to induce the whole transition are, in practice, unachievable due to the enhanced brittleness of the studied material. The recoverable strain in the martensite phase (about 3\%) is comparable to the Ni-Fe-Al ribbons [7]. 

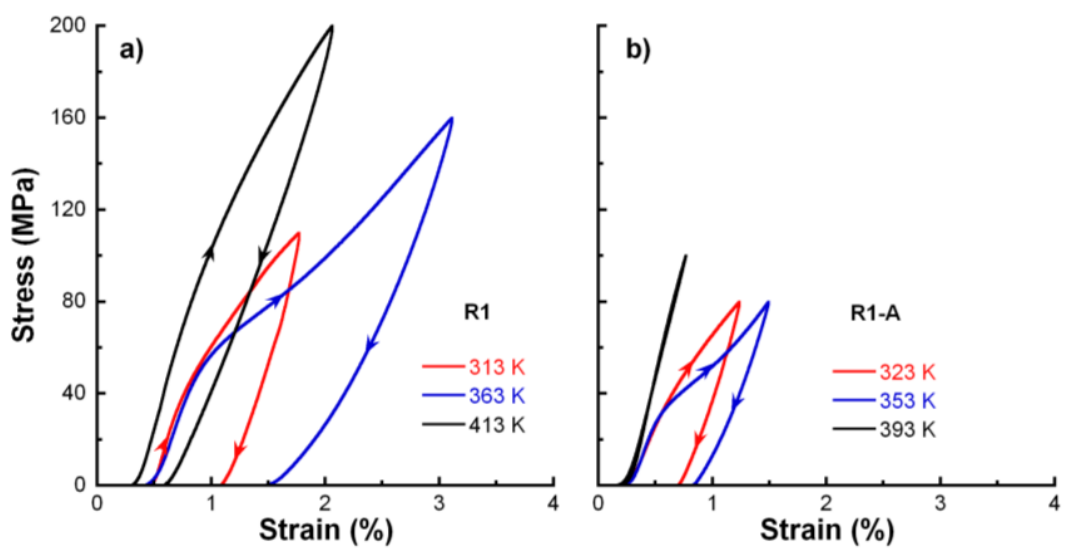

Figure 4. Tensile stress- strain curves at constant temperatures for Ni-Fe-Ga in as-cast state (a) and annealed at $523 \mathrm{~K}$ for $10 \mathrm{~min}$ (b).

In the austenite phase, increasing the temperature produces a reduction of the strain recovery (below 2\%) but larger stresses can be applied. The stress-strain measurements on the R1-A sample, Fig. 4(b), have been performed in the reduced stress interval to demonstrate ability for these materials to work at low level of the tensile stress. Indeed, Fig. 4(b) shows some superelastic strain recovery (about $0.8 \%$ ) in the temperature range of MT. As in the case of the as-cast ribbons, at temperatures below $\mathrm{A}_{\mathrm{F}}$, the transformation hysteresis prevents the complete reverse transformation, resulting in the unclosed loops; however, the remaining strain vanishes upon heating above $A_{F}$. Above MT, the specimen shows expected elastic behavior, with closed and anhysteretic loading-unloading $\sigma-\varepsilon$ curves depending on the test temperature.

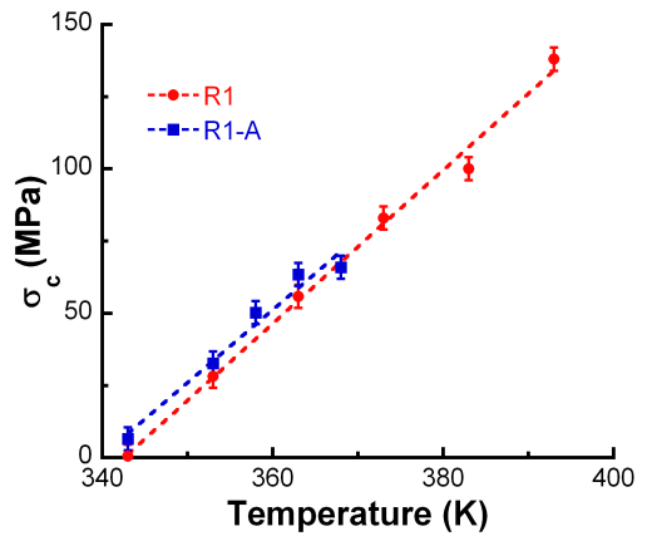

Figure 5. Critical stresses determined from the $\sigma-\varepsilon$ curves of the as-cast, R1, (a) and annealed ribbons, R1-A, (b) as a function of temperature. Dotted lines represent linear fits.

We have determined the diagram phase for the critical stress, $\sigma_{\mathrm{C}}$, at which the forward MT starts in the R1 and R1-A samples (see Fig. 5). Due to the smooth shape of $\sigma$ $\varepsilon$ curves, we have defined the numerical values of the critical stress by a tangent method, i.e., at the cross points of the linearly extrapolated initial slope and the slope during transformation [7]. The data in Fig. 5 have been approximated by the straight lines with slopes of 2.7 and 2.5 $\mathrm{MPa} / \mathrm{K}$ for $\mathrm{R} 1$ and $\mathrm{R} 1-\mathrm{A}$ samples respectively. The linear temperature dependence of $\sigma_{\mathrm{C}}$ in both R1 and R1A is typical of stress-induced MTs $[1,6]$.
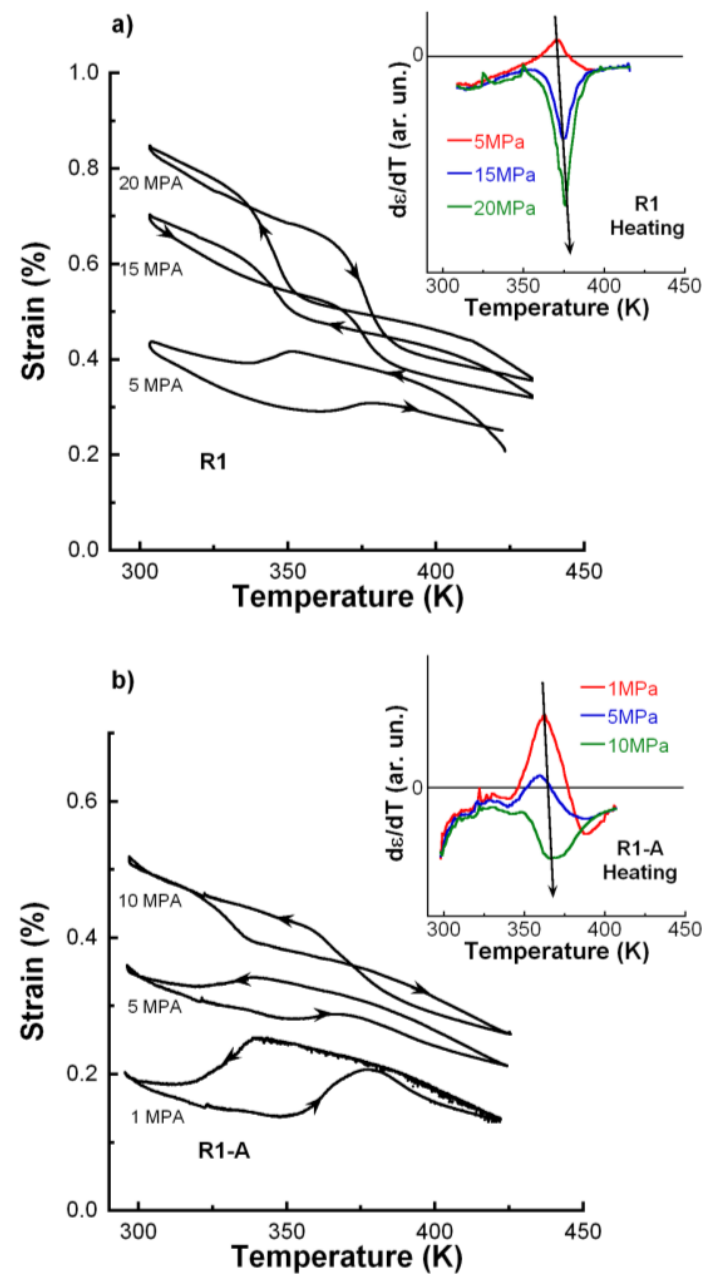

Figure 6. Strain versus temperature under constant loads recorded for the as-cast (a) and annealed at $523 \mathrm{~K}$ for $10 \mathrm{~min}$. (b) Ni-Fe-Ga ribbons. Insets show the temperature derivative of the strain as a function of the temperature for the respective heating runs reflecting influence of stress on the transformation temperature.

Figure 6 shows the strain versus temperature under constant loads up to $20 \mathrm{MPa}$ for the as-cast and annealed $\mathrm{Ni}-\mathrm{Fe}-\mathrm{Ga}$ ribbons. The curves for the as-cast and heattreated ribbons are characteristic of materials exhibiting a two-way shape memory effect by contraction/elongation during the forward and reverse martensitic 
transformations, which is accompanied by a thermal hysteresis. Note, however, that both specimens under tensile loading below a critical stress (15 $\mathrm{MPa}$ and 10 $\mathrm{MPa}$ for the as-cast and annealed ribbons respectively) suffer an anomalous contraction of the length at the forward MT instead of an elongation, and an extension at the reverse MT instead of the expected contraction. This behavior indicates the existence of a compressive internal stress alongside the ribbon length induced during the rapid solidification process. For the as-received specimens, the average internal stress could be accepted as $\sim 15 \mathrm{MPa}$, see above. A heat treatment, even during the short annealing time, naturally leads to a certain release of internal stresses, which produces a reduction of the average internal stress to about $10 \mathrm{MPa}$ in $\mathrm{R} 1-\mathrm{A}$ ribbon.

From the minimums of the $\mathrm{d} \varepsilon / \mathrm{d} T$ versus temperature curves (see insets in Fig. 6) we can estimate the behavior of $\mathrm{T}_{\mathrm{A}}(\sigma)$ for R1 and R1-A specimens. In each inset, the arrow joints the minimum of the curves, which indicates that the applied stress almost linearly shifts the MT towards higher temperatures. $\mathrm{T}_{\mathrm{M}}(\sigma)$ follows a similar trend, as observed from the data corresponding to the cooling runs at constant loads (not shown).

\subsubsection{Ni-Mn-Sn as-cast ribbons}

For the Ni-Mn-Sn ribbons, its high brittleness reduces the mechanical and thermomechanical analysis in tensile conditions to lower stresses. The isothermal stress-strain curves, depicted in Fig. 7 for temperatures below, during and above MT, show anomalous loops and no evidence of the plateau-like behavior characteristic of the superelasticity, at least for the maximum stress of 40 $\mathrm{MPa}$. Note that those curves at temperatures corresponding to the austenite phase present elastic features with positive values of the strain up to $0.4 \%$ corresponding to ribbon extension. For temperatures below $416 \mathrm{~K}\left(\mathrm{M}_{\mathrm{F}}\right)$, the strain is becoming negative (contraction) after removal of stress, indicating a contraction of the ribbon compared with its length at 523 $\mathrm{K}$ (remember that after each isothermal measurement, the sample was heated up to $523 \mathrm{~K}$ ). In the temperature range corresponding to forward MT, the curves exhibit extremely anomalous particular loop: low stresses in the load cycle enlarge the ribbons up to some value, and when transformation austenite-martensite starts a further increase of the stress up to $\sigma \sim 15 \mathrm{MPa}$ decreases the specimen length, then the ribbon expands again. In the unloading curve, the strain decreases almost linearly. This feature means that the longer-length ribbon in austenitic phase transforms into a shorter-length martensite phase under combined influence of applied tensile and the internal compressive stresses.

Figure 8 plots the $\varepsilon-T$ curves at constant loads for R2. The ribbons present a good shape memory effect, i.e., a reversible change in length associated to MT. Besides, the curves display the same anomalous behavior as previous Ni-Fe-Ga samples associated to the internal stresses: at low tensile constant loads applied in austenite, the specimen contracts during the forward transformation and elongates during the reverse transition. The tensile stress needed to turn over the effect of the internal stresses is $\sim 20 \mathrm{MPa}$, larger than for the Ni-Fe-Ga alloys. As Wang and co-workers have proposed [11], these larger internal stresses would be a consequence of the highly-oriented columnar grains observed in the ribbons, as occurs in our specimens.

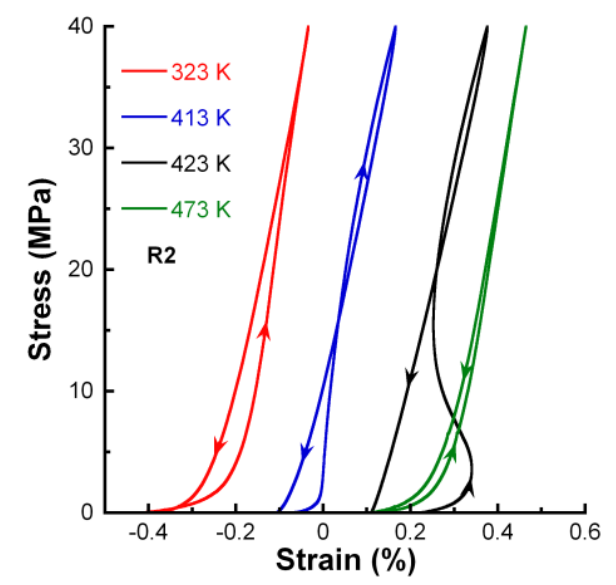

Figure 7. Tensile stress - strain curves registered in isothermal conditions at increasing temperature from $323 \mathrm{~K}$ to $473 \mathrm{~K}$ for as-cast Ni-Mn-Sn ribbons.

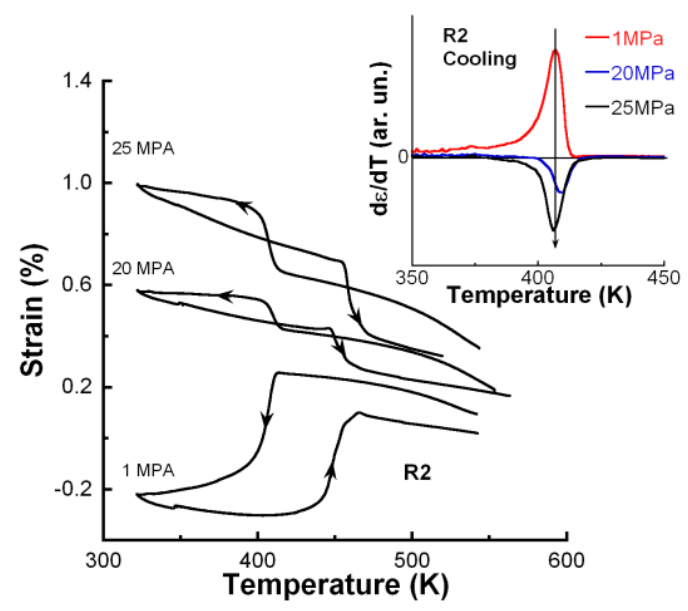

Figure 8. Strain-temperature dependences under constants loads registered for the Ni-Mn-Sn as-cast sample. Inset: temperature derivative of the strain as a function of the temperature for the cooling run.

Inset in Fig. 8 show plots of the temperature dependence of $\mathrm{d} \varepsilon / \mathrm{d} T$ obtained in the cooling run; in contrast to Ni-FeGa ribbons, the transition temperatures of R2 are nearly stress-independent, as weak changes in the location of the maxima and minima are observed.

\section{Summary}

The transformation and shape memory behaviors of the $\mathrm{Ni}_{55} \mathrm{Fe}_{16} \mathrm{Ga}_{29}$ (at.\%) and $\mathrm{Ni}_{50} \mathrm{Mn}_{40} \mathrm{Sn}_{10}$ (at.\%) ribbons have been studied in this work. While annealing of NiFe-Ga at $773 \mathrm{~K}$ eliminates MT, the one at $523 \mathrm{~K}$ leads to some shift of the martensitic transformation to larger temperatures and reduction of the internal stress. In the case of Ni-Mn-Sn ribbons, heat treatments enlarge their 
brittleness to the extent of impossibility to perform any reasonable (thermo-)mechanical testing .

$\mathrm{Ni}-\mathrm{Fe}-\mathrm{Ga}$ as-cast and annealed ribbons present nonlinear hysteretic $\sigma-\varepsilon$ curves below $\mathrm{A}_{\mathrm{F}}$ and, close to the MT, a temperature-dependent change in slope of the loading due to the induction of the phase transition by the stress.

An appreciable recovery of strain due superelasticity effect was also observed. Above enough $\mathrm{A}_{\mathrm{F}}$, the ribbons exhibit an elastic anhysteretic behavior. Besides, both samples show a two-way shape memory effect occurring at MT, characterized by a reversible length contraction/elongation of the specimens.

$\sigma-\varepsilon$ curves for Ni-Mn-Sn ribbons present modest plasticlike behavior, excepting at temperatures close to MT, at which low tensile stresses in the load cycle first elongate the ribbons followed by a contraction and an expansion for stresses above $15 \mathrm{MPa}$. This anomalous behavior indicates an interplay between internal compressive and external tensile stresses during the stress-induced phase transition from the austenite to the martensite.

The stress-strain behavior of studied Ni-Fe-Ga ribbons show their potential for elastocaloric applications. Considering the same objective, the mechanical properties of Ni-Mn-Sn ribbons must be improved.

\section{Acknowledgements}

This work has been carried out with the financial support of Projects No. CB-2010-01-157541 (CONACYT, Mexico), MAT2014-56116-C4-3-4-R (Spanish Ministry of Economy and Competitiveness), IT711-13 (Basque Government, Spain) and Laboratorio Nacional de Investigaciones en Nanociencias y Nanotecnología (LINAN,IPICyT). P.A. is grateful to Basque Government for financial support. Technical support from M. Sc. A. I. Peña Maldonado and M. Sc. B. A. Rivera-Escoto is acknowledged.

\section{References}

1. Shape Memory Alloys, Springer US, Boston, MA, 2008. Editor: D.C. Lagoudas.

2. B. Lu, F. Xiao, A. Yan, J. Liu, Elastocaloric effect in a textured polycrystalline Ni-Mn-In-Co metamagnetic shape memory alloy, Appl. Phys. Lett. 105 (2014) 161905.

3. L. Mañosa, S. Jarque-Farnos, E. Vives, A. Planes, Large temperature span and giant refrigerant capacity in elastocaloric $\mathrm{Cu}-\mathrm{Zn}-\mathrm{Al}$ shape memory alloys, Appl. Phys. Lett. 103 (2013) 211904. doi:10.1063/1.4832339.

4. R. Millán-Solsona, E. Stern-Taulats, E. Vives, A. Planes, J. Sharma, A.K. Nayak, et al., Large entropy change associated with the elastocaloric effect in polycrystalline $\mathrm{Ni}-\mathrm{Mn}$-Sb-Co magnetic shape memory alloys, Appl. Phys. Lett. 105 (2014) 241901. doi:10.1063/1.4904419.

5. P.O. Castillo-Villa, L. Mañosa, A. Planes, D.E. Soto-Parra, J.L. Sánchez-Llamazares, H. FloresZúñiga, et al., Elastocaloric and magnetocaloric effects in Ni-Mn-Sn(Cu) shape-memory alloy, J Appl. Phys. $113 \quad$ (2013) 053506. doi:10.1063/1.4790140.

6. A. Kosogor, V.A. L'vov, V.A. Chernenko, E. Villa, J.M. Barandiaran, T. Fukuda, et al., Hysteretic and anhysteretic tensile stress-strain behavior of $\mathrm{Ni}-\mathrm{Fe}(\mathrm{Co})-\mathrm{Ga}$ single crystal: Experiment and theory, Acta Mater. 66 (2014) 79-85.

7. V.A. Chernenko, B.R. Kanth, P.K. Mukhopadhyay, S.N. Kaul, E. Villa, A. Gambardella, et al., Stress-induced and thermoelastic properties of $\mathrm{Ni}-\mathrm{Fe}-\mathrm{Al}$ melt-spun ribbon, Appl. Phys. Lett. 93 (2008) 141904.

8. V.A. Chernenko, E. Cesari, J. Pons, C. Segui, Phase transformations in rapidly quenched $\mathrm{Ni}-$ Mn-Ga alloys, J. Mater. Res. 15 (2000) 14961504.

9. J. Rodríguez-Carvajal, Recent advances in magnetic structure determination by neutron powder diffraction, Phys. B Condens. Matter. 192 (1993) 55-69.

10. T. Krenke, M. Acet, E.F. Wassermann, X. Moya, L. Mañosa, A. Planes, Martensitic transitions and the nature of ferromagnetism in the austenitic and martensitic states of $\mathrm{Ni}-\mathrm{Mn}$ - Sn alloys, Phys. Rev. B. 72 (2005).

11. W. Wang, J. Yu, Q. Zhai, Z. Luo, H. Zheng, Origin of retarded martensitic transformation in Heusler Ni-Mn-Sn melt-spun ribbons, Intermetallics. 42 (2013) 126-129.

12. H.J. Yu, X.T. Zu, H. Fu, X.Y. Zhang, Z.G. Wang, Effect of annealing and heating/cooling rate on the transformation temperatures of $\mathrm{NiFeGa}$ alloy, J. Alloys Compd. 470 (2009) 237-240.

13. J.B. Lu, H.X. Yang, H.F. Tian, L.J. Zeng, C. Ma, L. Feng, et al., Cooperative effect of monoclinic distortion and sinusoidal modulation in the martensitic structure of Ni2FeGa, J. Solid State Chem. 183 (2010) 425-430.

14. F. Alvarado-Hernandez, D.E. Soto-Parra, R. Ochoa-Gamboa, P.O. Castillo-Villa, H. FloresZuniga, D. Rios-Jara, Thermal and structural study of $\mathrm{Ni}-\mathrm{Fe}-\mathrm{Ga}$ ferromagnetic shape memory alloys, J. Alloys Compd. 462 (2008) 442-445. 